\title{
Effect of Cigarette Smoke Components on Vascular Smooth Muscle Cell Migration Toward Platelet-Derived Growth Factor BB
}

\author{
Shinji Yoshiyama ${ }^{1}$, Takahiro Horinouchi ${ }^{2}$, Soichi Miwa ${ }^{2}$, Hong Hui Wang ${ }^{1}$, Kazuhiro Kohama ${ }^{1}$, \\ and Akio Nakamura ${ }^{1, *}$ \\ ${ }^{1}$ Department of Molecular and Cellular Pharmacology, Gunma University Graduate School of Medicine, \\ Gunma 371-8511, Japan \\ ${ }^{2}$ Department of Cellular Pharmacology, Hokkaido University Graduate School of Medicine, Hokkaido 060-8638, Japan
}

Received November 1, 2010; Accepted February 10, 2011

\begin{abstract}
Cigarette smoking is one of the factors causing accumulation of vascular smooth muscle cells (VSMCs) in atherosclerotic plaques. Changes in cell migration toward platelet-derived growth factor BB were investigated using a Boyden chamber after 48-h preincubation of GBaSM-4 VSMCs with nicotine or nicotine-free cigarette smoke extract (CSE). A nicotine concentration of $0.1 \mu \mathrm{M}$ maximally promoted cell migration; $0.1 \%$ CSE also promoted cell migration, while high CSE concentrations damaged GBaSM-4 cells. Fetal bovine serum (FBS) long-depletion induced decrease in migration of GBaSM-4 cells. Our results suggest that nicotine and some CSE components can induce GBaSM-4 cell migration.
\end{abstract}

Keywords: smooth muscle, nicotine, cigarette smoke extract (CSE)

Cigarette smoking is well-recognized as an etiology for many diseases and is considered to be one of the risk factors for arteriosclerosis (1). In atheromatous plaque formation, vascular smooth muscle cells (VSMCs) migrate from the tunica media toward the tunica intima where they proliferate and undergo phenotypic changes (2). The mechanisms of plaque formation development are not yet fully understood.

Nicotine is one of the most active pharmacological compounds in cigarette smoke. The mechanism of nicotine action on neuronal cells has been established, while the mechanism of direct influence of nicotine on VSMCs remains unknown. However, it has recently been reported that nicotine exhibits a direct effect on VSMCs $(3-5)$. We have also reported on the effect of nicotine as a chemoattractant for induction of migration of GBaSM-4 cells isolated from the basilar arteries of guinea pigs (6) and elucidated that nicotine acts through nicotinic acetylcholine receptors (7). However, up to now, no details about the functions of nicotinic acetylcholine receptors in VSMCs have been reported. On the other hand, cigarette smoke extract (CSE) is an established model for

*Corresponding author. gacho@med.gunma-u.ac.jp Published online in J-STAGE on March 16, 2011 (in advance) doi: $10.1254 /$ jphs.10283SC studying the comprehensive effects of cigarette smoke. CSE contains many chemical compounds; it possesses cytotoxicity against VSMCs and also enhanced the mitogenic effect in the presence of hydrogen peroxides $(8,9)$. We used CSE without nicotine in the present study.

VSMCs of chronic cigarette smokers are constantly exposed to nicotine and compounds in CSE. Nicotine and CSE may not show instantaneous effects on VSMCs; however, they possibly have long-term effects. Using a model of cell migration toward platelet-derived growth factor BB (PDGF-BB), we explored the effects of nicotine and CSE on VSMC migration.

The quantification of cell migration was performed using a Boyden chamber (Neuro Probe, Inc., Gaithersburg, $\mathrm{MD}, \mathrm{USA}$ ) according to the procedure described by $\mathrm{Li}$ et al. $(7,10)$. After preincubation with the testing compounds, the cells applied to the Boyden chamber were incubated for $4 \mathrm{~h}$ in a $\mathrm{CO}_{2}$ incubator. The cells that migrated through the filter were then stained using Giemsa's staining solution (Kanto Chemical Co., Inc., Tokyo) and counted in 5 fields per well under a magnification of $400 \times$.

Cell growth and cell viability were estimated by the 3-(4,5-dimethylthiazol-2-yl)-2,5-diphenyltetrazolium bromide (MTT) colorimetric assay according to the technique described by Mosmann (11). In brief, the cells 
were incubated with $0.5 \mathrm{mg} / \mathrm{ml} \mathrm{MTT}$ for $3 \mathrm{~h}$ in a $\mathrm{CO}_{2}$ incubator. Residual MTT was removed and MTT formazan was dissolved in $100 \mu$ l of dimethyl sulfoxide. The absorbance of MTT formazan at $570 \mathrm{~nm}$, which was directly proportional to viable cell number, was measured spectrophotometrically.

CSE was prepared from commercial cigarettes (HighLite; Nihontabakosangyo, Inc., Tokyo) according to the procedure described by Yamaguchi et al. (12). CSE concentration obtained from 4 cigarettes per $1 \mathrm{ml}$ of phosphate-buffered saline was defined as 100\% CSE.

Cell migration and proliferation were measured to determine the optimum fetal bovine serum (FBS) concentration for preincubation in the migration assay. GBaSM- 4 cells were preincubated with $0 \%-10 \%$ FBS for $48 \mathrm{~h}$ before the cell migration assay. The preincubation period was determined by referring to the periods employed in other nicotine exposure studies $(3,4)$. Relative cell migrations in $0 \%, 0.1 \%$, and $1 \%$ FBS were found to be reduced to $0 \%, 4.5 \pm 3.0 \%$, and $26.4 \pm 2.1 \%$, respectively, compared to cell migration in $10 \%$ FBS (Fig. 1A). For measuring cell proliferation, GBaSM-4 cells were incubated with $0 \%-10 \%$ FBS for 4 days. The relative cell proliferation was normalized to the number of seeded cells. Figure 1B shows that relative cell proliferation decreased as FBS concentration decreased and that proliferation was arrested in the absence of FBS. The results showed that it was difficult to estimate cell migration in the Boyden chamber for FBS concentrations below $0.1 \%$. We determined that $1 \%$ FBS was the optimum concentration for preincubation.

GBaSM-4 cells were preincubated with $0-10 \mu \mathrm{M}$ nicotine (Sigma-Aldrich Corp. St. Louis, MO, USA) for $48 \mathrm{~h}$ to examine the effect of nicotine on cell migration. The cells were divided into a positive migration group with PDGF-BB (Sigma-Aldrich Corp.) and a random migration group without PDGF-BB and placed into the Boyden chamber. Relative cell migration was calculated by [positive migration] / [random migration]. The relative migrations of cells preincubated with $0.01,0.1,1$, and 10 $\mu \mathrm{M}$ nicotine were $271 \pm 11 \%, 418 \pm 96 \%, 249 \pm 23 \%$, and $212 \pm 11 \%$, respectively, compared to migrations with $0 \mu \mathrm{M}$ nicotine (Fig. 2A). The promotion of cell migration by nicotine was not dose-dependent and this effect decreased for nicotine concentrations greater than $0.1 \mu \mathrm{M}$. This decremental effect might have been caused by cell damage during preincubation with higher concentrations of nicotine; we thus performed an MTT assay to confirm the viability of GBaSM-4 cells exposed to nicotine. GBaSM-4 cells were incubated with various concentrations of nicotine and their viability was measured after $0.5,2,12$, and $48 \mathrm{~h}$. Cell viability was not affected by nicotine concentration or incubation time (Fig. 2B).

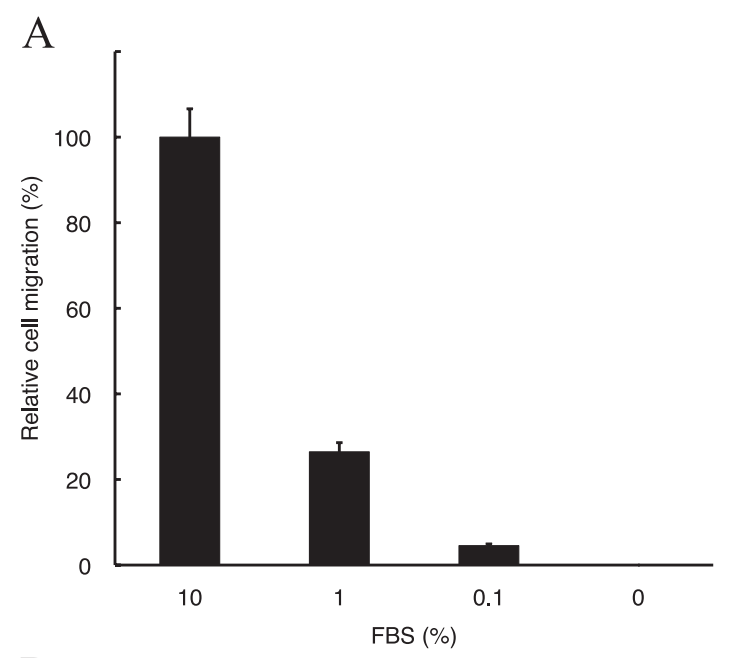

B

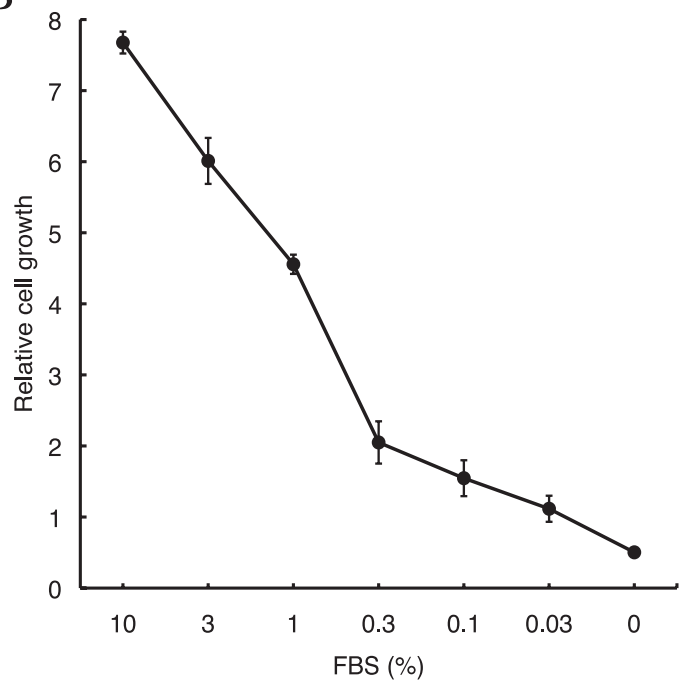

Fig. 1. FBS deprivation decreased cell migration and proliferation of GBaSM-4 cells. Cells were grown on a surface of Dulbecco's modified Eagle's medium (DMEM; Invitrogen Corp., Carlsbad, CA, USA) supplemented with $10 \%$ FBS and 50 units $/ \mathrm{ml}$ penicillin and $50 \mu \mathrm{g} / \mathrm{ml}$ streptomycin as antibiotics (Invitrogen). The cell culture was maintained at $37^{\circ} \mathrm{C}$ in a humidified atmosphere of $5 \% \mathrm{CO}_{2}$ using a $\mathrm{CO}_{2}$ incubator. A: The cells were preincubated for $48 \mathrm{~h}$ with $0 \%, 0.1 \%$, $1 \%$, and $10 \%$ FBS. The cells were suspended in DMEM containing $0.2 \%$ bovine serum albumin (BSA). The cell suspension $\left(8 \times 10^{3}\right)$ was placed on the upper well of the Boyden chamber. As a chemoattractant, $10 \mathrm{ng} / \mathrm{ml}$ PDGF-BB was added to DMEM containing $0.2 \%$ BSA in the bottom well. The $\mathrm{x}$-axis indicates the FBS concentration during preincubation. Data were presented as the percentage of $10 \%$ FBS \pm S.E.M. B: cells $\left(1 \times 10^{3}\right)$ were seeded in a 96-well microplate and cultured for 4 days in DMEM with $0 \%-10 \%$ FBS. Data were normalized to a value of $1 \times 10^{3}$ cells and presented as the foldchange \pm S.E.M.

We thus inferred that maximum relative cell migration occurred at $0.1 \mu \mathrm{M}$ nicotine. Our data is consistent with a previous report on cells from the thoracic aorta using $10 \% \mathrm{FBS}$ as a chemoattractant (3). Given that the plasma 
A

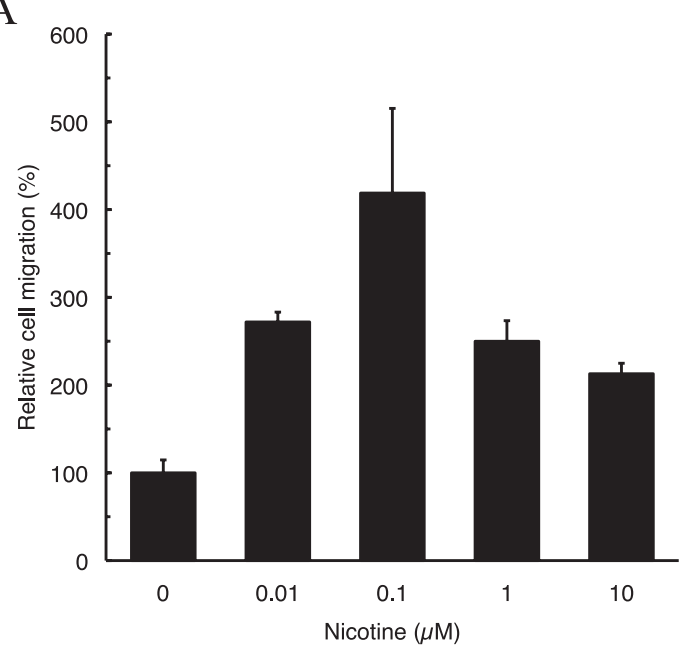

B

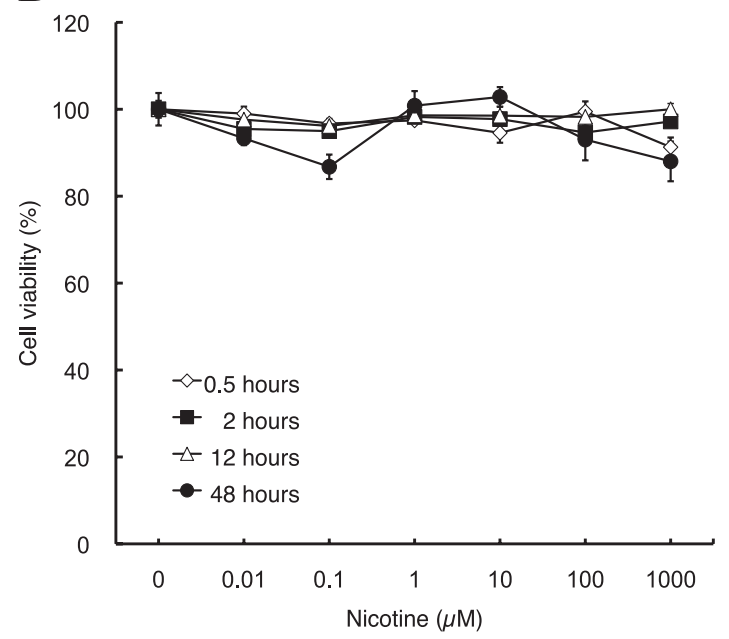

Fig. 2. Effect of preincubation with nicotine on GBaSM-4 cell migration and cell viability. The cells were cultured in DMEM containing $1 \% \mathrm{FBS}$ for $24 \mathrm{~h}$ prior to usage in experiments. A: After preincubation with $0-10 \mu \mathrm{M}$ nicotine for $48 \mathrm{~h}$, the cell suspension $\left(1 \times 10^{4}\right)$ was placed in the Boyden chamber. As a chemoattractant, $10 \mathrm{ng} / \mathrm{ml}$ PDGF-BB was added to DMEM containing $0.2 \%$ BSA in the bottom well. Data were expressed as the percentage of $10 \%$ FBS \pm S.E.M. B: cells $\left(5 \times 10^{3}\right)$ were seeded in a 96-well microplate. The cells were incubated from 0.5 to $48 \mathrm{~h}$ in DMEM containing 1\% FBS and at concentrations of nicotine ranging from 0 to $1000 \mu \mathrm{M}$. Data were presented as the percentage relative to the values in the absence of nicotine \pm S.E.M.

nicotine concentration in smokers ranges between 0.01 and $1 \mu \mathrm{M}(13)$, cell migration of VSMCs might be promoted by nicotine in vivo. We speculated that this promotive effect of nicotine is related to autocrine growth factors. Cucina et al. have already reported that nicotine promotes the secretion of PDGF, basic fibroblast growth factor (bFGF), and transforming growth factor- $\beta 1$ (13, 14). Kanda et al. also showed that nicotine promotes the secretion of epidermal growth factor (4). Figure 1A shows that the migration of GBaSM-4 cells toward
PDGF-BB was suppressed by incubation with $1 \%$ FBS compared with their migration by incubation with $10 \%$ FBS. The various growth factors in FBS regulate the phenotype of VSMCs. The promoting secretion of PDGF and bFGF from VSMCs by nicotine are bell-shaped patterns $(13,14)$, which resembled the pattern by nicotine. Thus, if GBaSM- 4 cells are stimulated by the autocrine secretion of growth factors in addition to the growth factors in FBS, we speculate that the suppression of migration can be relieved.

We also examined the effect of CSE on cell migration and viability. GBaSM-4 cells were preincubated with $0.01 \%-1 \%$ CSE for $48 \mathrm{~h}$. Relative cell migration was determined in the Boyden chamber in a manner similar to that done in the nicotine experiment. For preincubation with $0.01 \%, 0.1 \%$, and $1 \% \mathrm{CSE}$, the relative migration was $111 \pm 1 \%, 198 \pm 2 \%$, and $114 \pm 1 \%$, respectively, compared to that on preinubation with $0 \% \mathrm{CSE}$, and it was significantly enhanced only at $0.1 \%$ (Fig. $3 \mathrm{~A}$ ). We attempted to examine cell migration at higher concentrations, those above $1 \% \mathrm{CSE}$; however, cell migration was not detected at these concentrations (data not shown). GBaSM- 4 cells were incubated with $0 \%-8 \%$ CSE and cell viability was measured after $0.5,2,12$, and $48 \mathrm{~h}$ with an MTT assay. GBaSM-4 cells showed no damage by exposure to any concentration of CSE from $0 \%-8 \%$ for $0.5 \mathrm{~h}$. However, they showed signs of damage on exposure to higher CSE concentrations in this range when incubated for over $2 \mathrm{~h}$. Exposures to 1\% CSE and more for 12 and $48 \mathrm{~h}$ decreased cell viability to less than half that of the untreated cells (Fig. 3B). CSE shows many effects on VSMCs presumably because CSE is a mixture of many chemical compounds. The details of the various compounds in CSE are not completely known. CSE promoted GBaSM-4 cell migration toward PDGF-BB, but the peak value of relative migration was less than that by nicotine (Figs. 2A, 3A). The procedure for preparing CSE has not been standardized and is being continually refined by various groups. Currently, CSEs are prepared from different cigarette sources by different procedures. Thus, discussions citing findings from other papers must pay careful attention to these differences. Jing et al. described how CSE had a mitogenic effect on pulmonary artery smooth muscle cells and how proliferation was induced by low CSE concentrations (15). We speculate that the proliferative and mitogenic effects may have been induced by nicotine because CSE of Jing et al. included nicotine. However, CSE used in our study did not contain nicotine. We expect that CSE used in the present study included compounds that affected the proliferation and cell migration of GBaSM-4. The cytotoxicity of CSE against VSMCs is already known (8). As seen in Fig. 3B, GBaSM-4 cells in the cell migration assay should be 



Fig. 3. Effect of preincubation with CSE on GBaSM-4 cell migration and cell viability. Prior to experiments, the cells were cultured in DMEM containing $1 \%$ FBS for $24 \mathrm{~h}$. A: The cells were preincubated for $48 \mathrm{~h}$ at various concentrations of CSE. Cells $\left(1 \times 10^{4}\right)$ were placed in the Boyden chamber. See the legend for Fig. 2A for other details. B: cells $\left(5 \times 10^{3}\right)$ were seeded in a 96 -well microplate. They were incubated from 0.5 to $48 \mathrm{~h}$ in DMEM containing $1 \%$ FBS at various concentrations of CSE. Data were presented as the percentage relative to the values in the absence of CSE \pm S.E.M.

damaged during the preincubation with $1 \%$ CSE for 48 $\mathrm{h}$. The signal transduction pathway for the damage is now under investigation.

Our results support that both nicotine and nicotine-free CSE promoted GBaSM-4 cell migration toward chemoattractants such as PDGF-BB in vitro. We speculate that chronic cigarette smoking actually also induces cell migration of VSMCs in the tunica media toward the atheromatous plaque.

\section{Acknowledgments}

This work was supported by grants from the Smoking Research
Foundation and by Grants-in-Aid for Scientific Research from the Ministry of Education, Culture, Sports, Science, and Technology of Japan.

\section{References}

1 Jonas MA, Oates JA, Ockene JK, Hennekens CH. Statement on smoking and cardiovascular disease for health care professionals. Circulation. 1992;86:1664-1669.

2 Libby P. Current concepts of the pathogenesis of the acute coronary syndromes. Circulation. 2001;104:365-372.

3 Luozzo DL, Pradhan S, Dhadwal AK, Chen A, Ueno H, Sumpio ES. Nicotine induces mitogen-activated protein kinase dependent vascular smooth muscle cell migration. Atherosclerosis. 2005; 178:271-277.

4 Kanda Y, Watanabe Y. Nicotine-induced vascular endothelial growth factor release via the EGFR-ERK pathway in rat vascular smooth muscle cells. Life Sci. 2007;80:1409-1414.

5 Cucina A, Fuso A, Coluccia P, Cavallaro A. Nicotine inhibits apoptosis and stimulates proliferation in aortic smooth muscle cells through a functional nicotinic acetylcholine receptor. J Surg Res. 2008;150:227-235.

6 Bao J, Oishi K, Yamada T, Liu L, Nakamura A, Uchida MK, et al. Role of the short isoform of myosin light chain kinase in the contraction of cultured smooth muscle cells as examined by its down-regulation. Proc Natl Acad Sci U S A. 2002;99:95569561.

7 Li S, Zhao T, Xin H, Ye LH, Zhang X, Tanaka H, et al. Nicotinic acetylcholine receptor $\alpha 7$ subunit mediates migration of vascular smooth muscle cells toward nicotine. J Pharmacol Sci. 2004; 94:334-382.

8 Ambalavanan N, Carlo WF, Bulger A, Shi J, Philips III JB. Effect of cigarette smoke extract on neonatal porcine vascular smooth muscle cells. Toxicol Appl Pharmacol. 2001;170:130-136.

9 Nishino E, Watanabe Y. Cigarette smoke extract is a modulator of mitogenic action in vascular smooth muscle cells. Life Sci. 1998;62:1339-1347.

10 Harvath L, Falk W, Leonard EJ. Rapid quantitation of neutrophil chemotaxis: use of a polyvinylpyrrolidone-free polycarbonate membrane in a multiwell assembly. J Immunol Methods. 1980; 37:39-45.

11 Mosmann T. Rapid colorimetric assay for cellular growth and survival: application to proliferation and cytotoxicity assays. J Immunol Methods. 1983;65:55-63.

12 Yamaguchi Y, Matsuno S, Kagota S, Haginaka J, Kunitomo M. Oxidants in cigarette smoke extract heritable hyperlipidemic rabbits. Atherosclerosis. 2002;156:109-117.

13 Cucina A, Sapienza P, Corvino V, Borrelli V, Randone B, SantoroD'Angelo L, et al. Nicotine induces platelet-derived growth factor release and cytoskeletal alteration in aortic smooth muscle cells. Surgery. 2000;127:72-78.

14 Cucina A, Sapienza P, Corvino V, Borrelli V, Mariani V, Randone $\mathrm{B}$, et al. Nicotine-induced smooth muscle cell proliferation is mediated through bFGF and TGF- $\beta_{1}$. Surgery. 2000;127: 316-322.

15 Jing HU, Yong-jian XU, Zhen-xiang Z, Feng T. Effect of cigarette smoke extract on proliferation of rat pulmonary artery smooth muscle cells and the relevant roles of protein kinase C. Chin Med J. 2007;120:1523-1528. 